\title{
Fiber optic system to measure adulteration in liquids using an RGB detection
}

\author{
O. X. Vera-Duarte ${ }^{a}$, J. D. Filoteo-Razo ${ }^{a}$, J. M. Estudillo-Ayala ${ }^{a, *}$, J. C. Hernández-García ${ }^{a, b}$, D. Jauregui-Vázquez ${ }^{a}$, \\ J. M. Sierra-Hernández ${ }^{a}$, J. R. Martinez-Angulo ${ }^{c}$, J. A. Martin-Vela ${ }^{a}$ and R. Rojas-Laguna ${ }^{a}$ \\ ${ }^{a}$ División de ingenierías CIS, Universidad de Guanajuato, \\ Carretera Salamanca-Valle de Santiago Km 3.5+1.8 Km, \\ Comunidad de Palo Blanco, Salamanca, Gto., 36885, México. \\ *e-mail julian@ugto.mx \\ ${ }^{b}$ CONACYT. catedrático CONACYT, \\ Av. Insurgentes Sur No. 1582, Crédito Constructor, Benito Juárez, 39040, México. \\ ${ }^{c}$ Universidad Autónoma de Tamaulipas, Facultad de Ingeniería y Ciencias, \\ Ciudad Victoria, Tamaulipas, 87149, México.
}

Received 3 December 2020; accepted 2 February 2021

In this paper, we propose a fiber optic system to measure the changes in the RGB composition of light in liquid solutions using a plastic optical fiber (POF) as an intrinsic sensor. The measurement system is composed of a $3 \mathrm{~W}$ white light-emitting diode as the light source, a 20 $\mathrm{cm}$ long U-shaped POF, and an RGB photodiode array that allows us to detect the changes in the RGB composition of the light propagating through the POF. The U-shaped POF has a length of $5 \mathrm{~mm}$ without coating, which remains in contact with the liquid solutions. The tests were performed with different concentrations of a mixture of tequila and water. The results obtained using the RGB photodiode array showed that the system has a resolution of $0.39 \%$ and a color change sensitivity of $0.1,0.6$, and $0.48-$ bits $\%$ for red, green, and blue colors, respectively. These results can facilitate the design of a low-cost prototype to measure the amount of adulteration in liquids.

Keywords: Fiber optic; sensor; refractive index; RGB color

PACS: 42.81.-i; 85.60.-q; 42.15.-i

\section{Introduction}

Fiber optic-based sensors are widely used in the optical sensing of physical properties for quantitative estimation. These devices are lightweight and compact and allow the implementation of multiplexing schemes with other fiber optic elements [1]. Fiber optic cables are unaffected by electromagnetic interference while propagating a beam of light, unlike copper cables. A plastic optical fiber (POF) is a low-cost material used in the manufacture of optical sensors. Recent advances in polymer technology and its advantages, including improved material transparency, have made POF a viable alternative that can replace electronic devices [2,3]. This allows POF sensors to measure different types of physical properties, such as temperature, viscosity, concentration, and refractive index of a solution. Additionally, other POF sensors have been applied to measure changes in color intensity, ultra-violet radiation, temperature, food and fruit ripening [4-10].

Additionally, POFs are advantageous to the electronic devices used in the measurement of physical properties because they are unaffected by electromagnetic noise, can be operated at high bandwidth, yield low losses, and can be used in highly acidic environments and extreme temperatures [11,12]. POF has unique features, which make it suitable for application in fiber optic sensors as a signal transmitter (extrinsic sensor) or head sensor (intrinsic sensor). Owing to its large fiber diam- eter ( 0.5 to $2 \mathrm{~mm})$, the POF can be easily coupled with light sources, such as light-emitting diodes (LEDs) to develop lowcost optoelectronic devices [13].

Further, for application as an intrinsic sensor, the POF can be configured in several ways, such as in-line, U-shape, and gamma configurations. In the U-shape and gamma configurations, the length of the uncoated fiber and the bending diameter, with and without coating, are varied. It has been shown that a longer uncoated length and smaller bending diameter yield better sensitivity and resolution [14]. Another important feature of POF is its low loss of 0.15 to $0.2 \mathrm{~dB} / \mathrm{m}$ in the visible region [15].

For this reason, visible LEDs have been used as light sources with the POF. Therefore, we propose a fiber optic system to measure the changes in the RGB composition of light in liquid solutions while using a POF as an intrinsic sensor. The U-shaped POF together with the RGB photodiode array allows the detection of changes in the RGB composition of the light intensity propagating through the optical fiber. We verified this setup using different concentrations of a mixture of tequila and water. The results showed that our system had a resolution of $0.39 \%$ and color change sensitivity of $0.10,0.6$, and $0.48-$ bits $/ \%$ for red, green, and blue, respectively. These results can be employed to design a low-cost prototype to measure the amount of adulteration in liquids. 


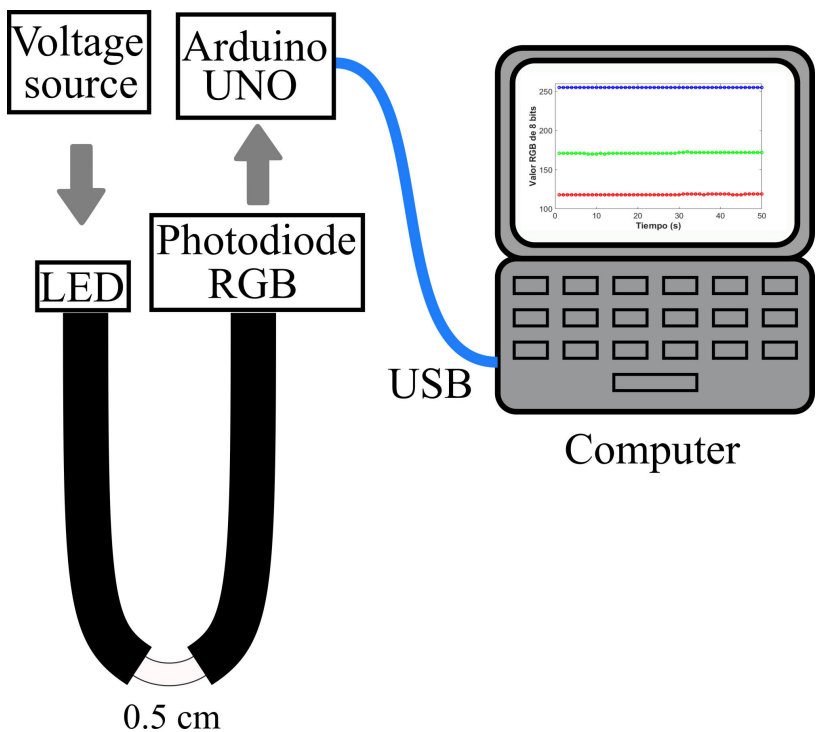

FIGURE 1. Schematic diagram of the fiber-optic system for measuring the changes in RGB composition.

\section{Experimental setup}

Figure 1 the schematic of the fiber optic system for measuring the changes in the RGB composition. The system comprises a $5 \mathrm{~V}$ voltage source to supply power to the $3 \mathrm{~W}$ LED. The light is transmitted through the U-shaped POF, which has a diameter of $5 \mathrm{~mm}$ without coating and large curvature (see Fig. 1). This section of the uncoated optical fiber enables contact with liquid solutions. The POF is made of polymethylmethacrylate resin with a core refractive index of 1.49 (numerical aperture $=0.5$ ) and has a core of $980 \mu \mathrm{m}$, coating diameter of $1000 \mu \mathrm{m}$, and an overall fiber diameter of $2000 \mu \mathrm{m}$. The transmitted light is received by the RGB photodiode array, which is connected to an Arduino UNO microcontroller and a computer via USB. The data is obtained by performing a measurement sweep. This data is sent to the computer to display the changes in the RGB composition via a graph.

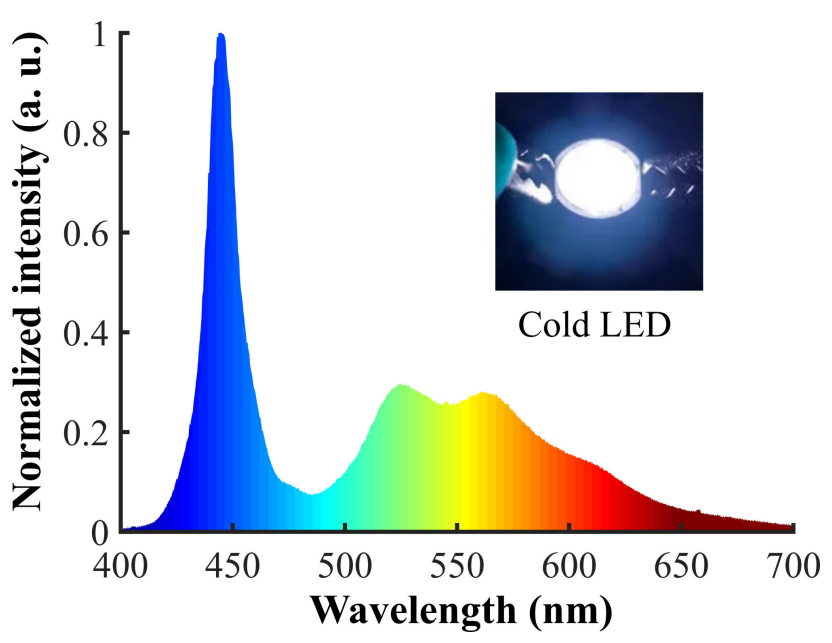

FIGURE 2. Spectrum of the LED used as a source of white light.

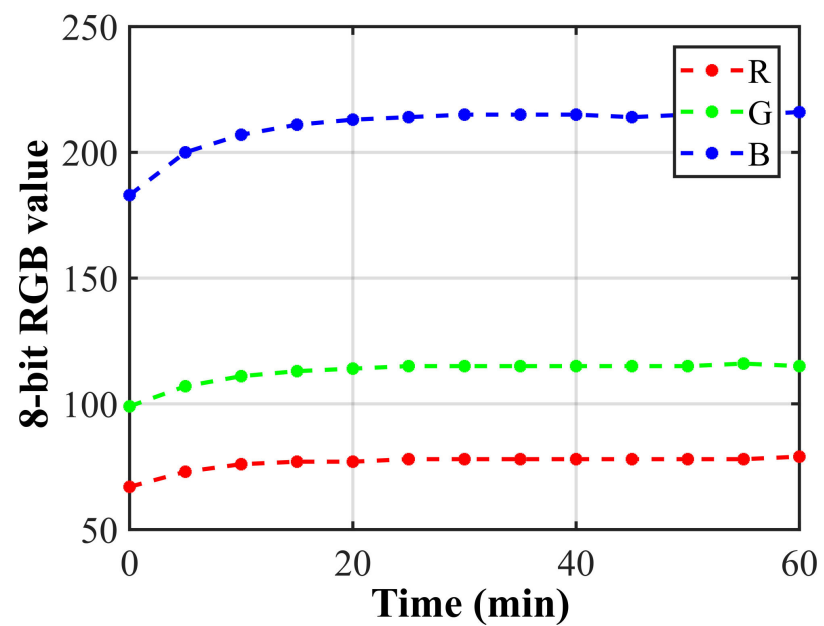

FIGURE 3. Stability test of a white light source when monitored with RGB photodiode array.

The white LED has a color temperature of $6500 \mathrm{~K}$ [16] with a spectrum of 400 to $700 \mathrm{~nm}$, as shown in Fig. 2. This spectrum was measured with an Ocean Optics model USB2000+ optical spectrum analyzer. The color temperature emitted by the LED makes it a cold white light source. Therefore, a peak is observed at a wavelength of $450 \mathrm{~nm}$, which corresponds to the blue color region of the visible spectrum.

Next, we tested the optical fiber system. First, a stability test was performed on the fiber optic system to observe whether the intensity of the white light source received by the RGB photodiodes remains stable during operation for a long time. Measurements were taken every 5 min during $1 \mathrm{~h}$ of operation. Figure 3 shows the variation of the 8-bit RGB color scale during the first $20 \mathrm{~min}$. This variation could be because of the temperature of the P-N junction of the power LED. However, when using the power LED, a higher applied voltage is required for operation; thus, more heat is generated. The system temperature during stability was $25^{\circ} \mathrm{C}$ (ambient temperature). After $20 \mathrm{~min}$, the RGB color scale became stable. Thus, we confirmed that the elements used in the fiber optic system work in optimal conditions.

Four samples of $50 \mathrm{ml}$ each were prepared with different tequila and water concentrations (Table I). For each sample (liquid solution), we conducted a sweep of 50 measurements of the RGB components. These were taken $20 \mathrm{~min}$ after the light source was turned on to ensure stable measurements.

TABLE I. Samples of solutions with percentage concentration.

\begin{tabular}{ccccc}
\hline $\begin{array}{c}\text { Sample } \\
\text { Liquid solution }\end{array}$ & $\mathrm{ml}$ & $\%$ & $\mathrm{ml}$ & $\%$ \\
\hline A & 5 & 10 & 45 & 90 \\
B & 20 & 40 & 15 & 60 \\
C & 35 & 70 & 10 & 30 \\
D & 50 & 100 & 0 & 0 \\
\hline
\end{tabular}




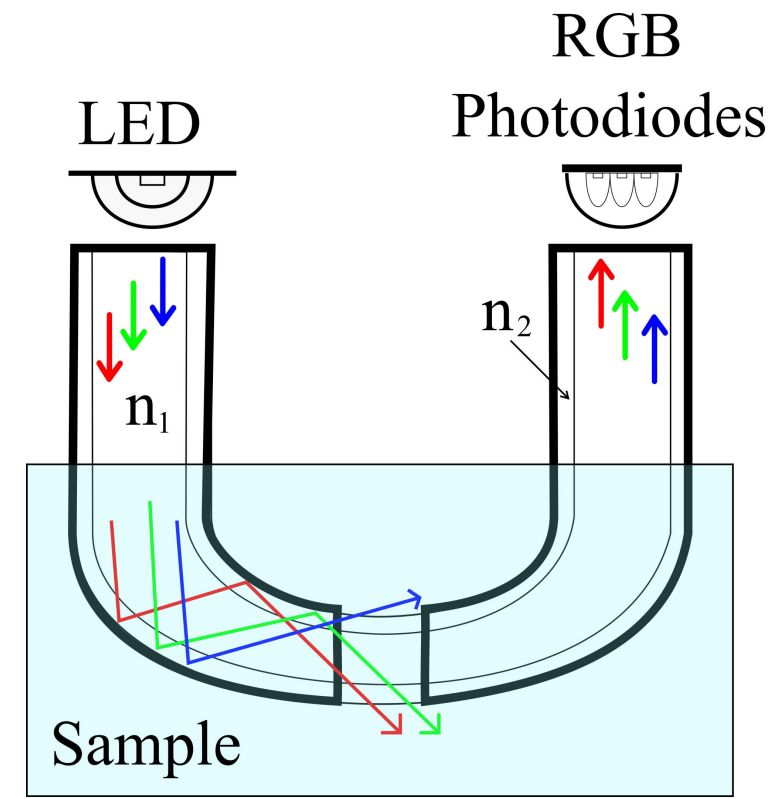

FIGURE 4. Samples of solutions with percentage concentration.

The solutions were analyzed using the fiber optic system. Each sample was measured 50 times to determine the average RGB color intensity.

\section{Operation principle}

The operating principle of the U-shaped POF is based on the attenuated total reflection (ATR) theory. This theory is based on the attenuation of the transmitted signal due to the losses suffered by the coupling of the evanescent field. Therefore, this causes the transmitted signal to continue to be guided by the optical fiber but is outside of it. The ATR uses the total internal reflection (TIR) property of light in which a small area of the uncoated optical waveguide allows light from the core to couple to the surrounding medium in order to interact with the sample (absorption) [17, 18]. Figure 4 shows a representation of the basic principle of an evanescent wave via ray tracing in a POF sensor in a U-shaped configuration. In this U-shaped configuration, due to the curvature, the power coupling and penetration depth of the evanescent field are increased, making it more sensitive to changes in the refractive index. The light received by the photodiodes is separated in each RGB color channel on an 8-bit scale (0 to 255). Using

TABLE II. Average RGB of the samples.

\begin{tabular}{cccc}
\hline Sample & R & G & B \\
\hline No sample & 72 & 106 & 198 \\
A & 66 & 97 & 176 \\
B & 69 & 99 & 181 \\
C & 72 & 105 & 194 \\
D & 75 & 110 & 206 \\
\hline
\end{tabular}

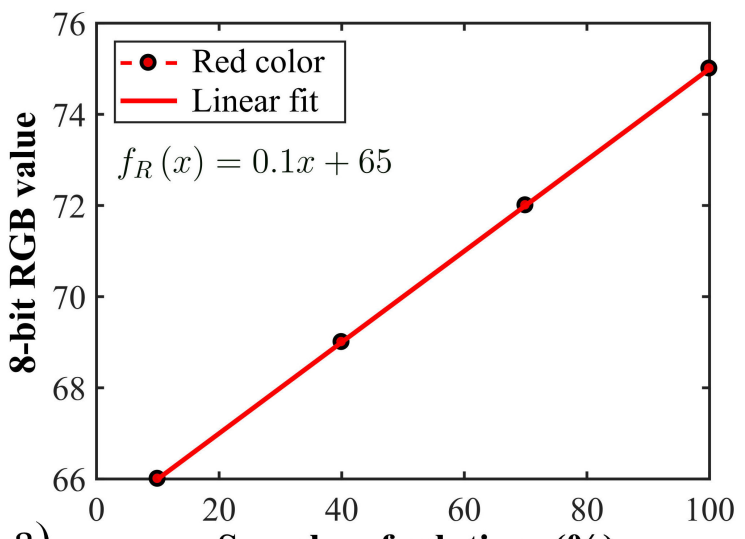

a)

Samples of solutions (\%)
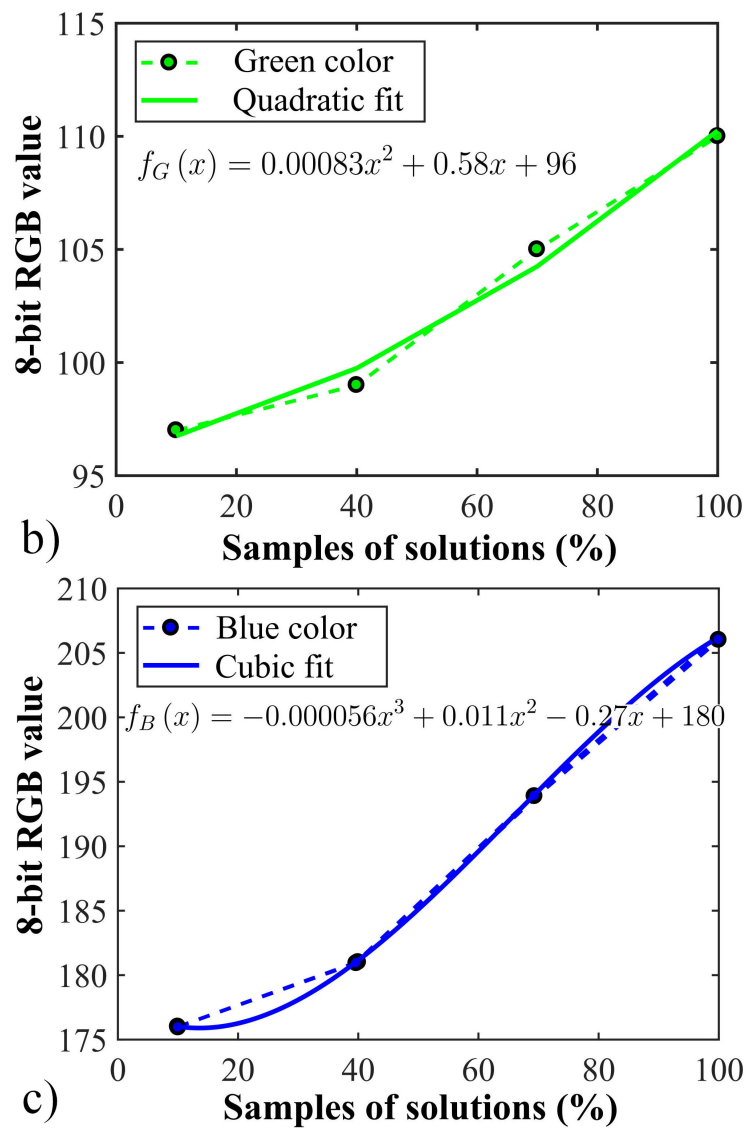

FIGURE 5. Values of RGB components in the samples A-D.

this 8-bit scale, each color channel shows 256 tones $(0+255)$ and a total of 16.7 million colors sent by the RGB photodiode array.

\section{Results and discussion}

Table II shows the RGB values (which range from 0 to 255) of the samples described in Table I. As can be observed in Table II, by increasing the percentage concentration of tequila and decreasing the percentage concentration of water, the RGB color values increase from 66 to 75,97 to 110 , and 176 to 206 , respectively. The maximum variations in the RGB color samples from the reference (no sample) are 9, 13, and 


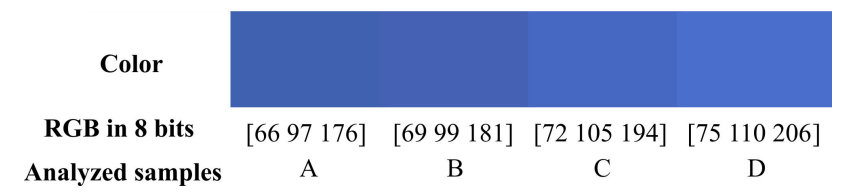

FIGURE 6. Color diagram of the analyzed samples A-D.

30 8-bits-result, respectively. Due to the peak at a wavelength of $470 \mathrm{~nm}$ in the white light source, the variation in blue is greater than that in other colors. Therefore, a greater change in blue was detected by the RGB photodiode array.

Using the results obtained in Table II, we can plot each component of the RGB colors of all the samples to observe the variation between each sample A-D in detail and plot a curve fit. This curve fitting allows us to establish an equation for the change in the percentages of concentration of water and tequila in each sample. Figure 5 shows the graphs corresponding to the results obtained in Table II. It can be observed that a higher concentration of tequila results in a higher value of the RGB components.

Other important parameters of the fiber optic system are the resolution and sensitivity of the POF intrinsic sensor. The resolution $(R)$ is the minimum change for which changes in a signal are noted and is determined by Eqs. (1) and (2).

$$
\begin{aligned}
A r & =\frac{A x}{2^{\# b i t s}} \\
R & =\frac{A r}{A x} \times 100 \%,
\end{aligned}
$$

where $A_{x}$ is the measurement range. In this case, it is 50 measurements per sample. $A_{r}$ is the value detected by the RGB photodiodes. Because the detected value is a number between 0 and 255, it is represented by 8 bits. From Eq. (5), $R=0.39 \%$. Sensitivity $(S)$ is the relationship between the increase in the output signal with respect to the increase in the measured variable. It is expressed as

$$
S=\left.\frac{\partial f_{R G B}(x)}{\partial x}\right|_{x \rightarrow x_{0}},
$$

where $f_{R G B}(x)$ is the equation of the curve fit of the RGB components. Substituting the curve fitting equations that are shown in Fig. 5 of each RGB component in Eq. (3), the following modified equations are obtained

$$
\begin{aligned}
& S_{R}=0.18 \text {-bits } / \% \\
& S_{G}=0.68 \text {-bits } / \% \\
& S_{B}=0.48 \text {-bits } / \%
\end{aligned}
$$

Finally, in Fig. 6, the color scale of the samples analyzed with the different concentrations of tequila and water is presented. Here, we observe the change in color level for different samples. A navy-blue color variation can be observed due to the LED spectrum. The peak at $470 \mathrm{~nm}$ affects the average of the colors in the RGB of the samples analyzed with the different concentrations, yielding a blue color. Besides for each RGB component, gradual variation in color is observed with increasing the concentration of tequila.

\section{Conclusions}

In this study, a fiber optic system was developed to measure RGB color changes in liquid solutions. This system was built with low-cost elements, such as a POF, power LED, and microcontroller. Owing to the different refractive indices of the POF and the solutions, we could observe RGB color changes. The results obtained from the samples containing tequila and water in different proportions showed high stability and consistency. We obtained a resolution of $0.39 \%$ and color change sensitivity of $0.1,0.6$, and 0.48 -bits $/ \%$ for red, green, and blue, respectively. The sensitivity can be further improved by increasing the length of the uncoated portion of the POF. Moreover, changing the cold-type LED to a warm-type LED can intensify the color changes in the RGB photodiode array. Finally, the results obtained and analyzed with the tequila and water samples would facilitate the design of a low-cost prototype for measuring the amount of adulteration in liquids in the future.

\section{Acknowledgments}

J. D. Filoteo-Razo was supported by the CONACYT grant No. 477506. This work was supported by the CONACYT projects Cátedras-CONACYT No. 3155, No. A1-S33363/CB2018; and by the University of Guanajuato through the Research and Postgraduate Support Department (DAIP) project No. CIIC 230/2020. J. M. Estudillo-Ayala recognizes his mentor Evgeny Kuzin, who was the pioneer in researchings of fiber optics in MEXICO.
1. C. Vázquez, A.B. Gonzalo, S. Vargas and J. Montalvo, Multisensor system using plastic optical fibers for intrinsically safe level measurements, Sensors Actuators A Phys. 116 (2004) 22, https://doi.org/10.1016/j.sna.2004.03.035

2. K.T.V. Grattan and T. Sun, Fiber optic sensor technology: An overview, Sensors Actuators A Phys. 82 (2000) 40, https:
//doi.org/10.1016/S0924-4247(99)00368-4

3. L. Bilro, N. Alberto, J.L. Pinto and R. Nogueira, Optical sensors based on plastic fibers, Sensors (Switzerland) 12 (2012) 2184, https://doi.org/10.3390/s120912184

4. M. Yunus and A. Arifin, Design of Oil Viscosity Sensor Based on Plastic Optical Fiber, J. Phys. Conf. Ser. 979 (2018) 012083, 
https://doi.org/10.1088/1742-6596/979/1/ 012083

5. J. D. Filoteo-Razo et al., RGB color sensor implemented with LEDs, Proc. SPIE - Int. Soc. Opt. Eng. (2015), https:// doi.org/10.1117/12.2188243

6. J. Zubia and J. Arrue, Plastic optical fibers: An introduction to their technological processes and applications, Opt. Fiber Technol. 7 (2001) 101, https://doi.org/10.1006/ofte. 2000.0355 .

7. J. D. Filoteo-Razo et al., Sensor RGB para detectar cambios de color en piel de frutas, Acta Univ. 26 (2016) 24, https : //doi.org/10.15174/au.2016.859

8. Y.S. Ong, E. Lewis, I. Grout and W. Mohammed, Results Classification in an RGB LED Based Optical Fiber Sensor System using Python, 2018 15th Int. Conf. Electr. Eng. Comput. Telecommun. Inf. Technol., IEEE (2018) 33-36, https: //doi.org/10.1109/ECTICon.2018.8619985

9. C. Novo et al., Plastic optical fibre sensor for quality control in food industry, Fifth Eur. Work. Opt. Fibre Sensors, International Society for Optics and Photonics (2013) 879411, https://doi.org/10.1117/12.2025489

10. D. Lopez-Betancur et al., Effects of Colored Light on Growth and Nutritional Composition of Tilapia, and Biofloc as a Food Source, Appl. Sci.10 (2020) 362, https://doi.org/10. $3390 /$ app10010362.

11. A. Arifin, Yusran, Miftahuddin, B. Abdullah and D. Tahir, Comparison of sensitivity and resolution load sensor at various configuration polymer optical fiber, AIP Conf. Proc., American Institute of Physics Inc. (2017), https://doi.org/ $10.1063 / 1.4973100$

12. F. Berghmans and H. Thienpont, Plastic optical fibers for sensing applications, Conf. Opt. Fiber Commun. Tech. Dig.
Ser. (2014), https://doi.org/10.1109/OFC.2014. 6886975

13. D.-S. Lee, M.Y. Jung, B.G. Jeon and M.-J. Sohn, Novel optical absorbance-based multi-analytes detection module using a tri-chromatic LED, PDs and plastic optical fibers and its application to a palm-sized urine test strip reader, Sensors 2010 IEEE (2010) 1411-1414, https://doi.org/10.1109/ ICSENS.2010.5690509

14. D.Z. Stupar et al., Remote monitoring of water salinity by using side-polished fiber-optic U-shaped sensor, 2012 15th Int. Power Electron. Motion Control Conf., IEEE (2012), LS4c-4, https://doi.org/10.1109/ EPEPEMC.2012.6397458

15. O. Ziemann, J. Krauser, P.E. Zamzow and W. Daum, POF handbook, Springer (2008), LS4c-4, https://doi.org/10. 1007/978-3-540-76629-2

16. I. Speier and M. Salsbury, Color temperature tunable white light LED system, Sixth Int. Conf. Solid State Light., International Society for Optics and Photonics (2006), 63371F, https://doi.org/10.1117/12.679688

17. S. F. Memon, E. Lewis, M. M. Ali, J. T. Pembroke and B. S. Chowdhry, U-bend evanescent wave plastic optical fibre sensor for minute level concentration detection of ethanol corresponding to biofuel production rate, 2017 IEEE Sensors Appl. Symp., IEEE (2017), 1-5, https://doi.org/10.1109/ SAS.2017.7894101

18. S. Wang, D. Zhang, Y. Xu, S. Sun and X. Sun, Refractive Index Sensor Based on Double Side-Polished U-Shaped Plastic Optical Fiber, Sensors 20 (2020) 5253, https: / / doi .org/10 . $3390 / s 20185253$ 